\title{
Specific training program for improving the shooting skills toward the goal in field hockey
}

\author{
* Mohamed Ahmed Mahmoud Ali Badr
}

Introduction

Field hockey is now a quick and strong sport, which can be observed during the movement of the players when receiving, passing and shooting the ball, also during the moving and changing playing positions the match play.

Match analysis is a technique for analyzing some important aspects such as technical and skill abilities of field hockey players, and becoming an increasingly integral part of the coaching process in elite sport (Hughes, 2004; Hughes \& Bartlett, 2002; Lythe, 2008). Monitoring field hockey players during match play to develop the most important technical skills is an important demand for players to scoring the goals during the field hockey matches (Sofwan et al., 2012).

Elite field hockey players during the game need high level technical skills such as being able to dribble without losing running speed and shooting to score goals. For a technically good player, dribbling and shooting are essentially an automatic process, and the better players distinguish themselves by their running speed while dribbling the ball and shooting during a rapid movements (Keogh et al., 2003).

Field hockey provides a constantly changing environment that challenges physical skill with the ball. Skilled performance is dictated by decision making and defined as the process by which a player uses a learned ability to organize, initiate and execute correct technique (Abernethy, 1996). It can be argued that player ability to hitting the ball toward the goal is important action during field hockey matches, while the player is inside the hitting circle.

The match analysis is a systematic process that aims to provide information for the purposes of conditioning and coaching. To be useful, performance analysis 
must support the coaching process and it requires a coupling of information between match observations and the training process (Lames \& Hansen, 2001). The fundamental characteristics of field sport performance such as field hockey can be obtained through analysis of the game. Lemmink et al. (2004) suggested that, the aspects of quantitative systematic analysis have been used to assist in making recommendations for tactical and skills-related strategies for both individual players and teams as a collective.

In addition, (Appleby \& Dawson, 2002; Spencer et al., 2004) suggested that, the results of objective performance analysis can enhance the coaching process, providing information and data to assist with decisions made about training, selection and talent identification.

In context of the relationship between the match analysis and the technical skills, (Elferink-Gemser et al., 2004) showed that the elite players fared better than the sub-elite players on technical and tactical variables and that elite players had developed these skills by the age of 14 .

Based on match analysis in 2014 Men's Hockey World Cup, the statistics reported 601 times the ball inside the hitting circle, and only 247 times the players hit the ball toward the goal, and from this numbers only 127 goals were on the target, however 120 shots weren't in the goal target. These match statistics found $48.58 \%$ of the total shots weren't on the goal target. In addition, there are only 72 goals scored from 127 shots on the goal target.

Thus, the lack of scoring the goal and the limiting of shooting numbers during the field hockey game had motived the researcher to investigate the zone area of shooting toward the goal target and to identify the most effective shooting skills, which cause the most goals scored.

In addition, there is limited scientific research into field hockey in Egypt, especially in the match analysis demands, which are important requirements for success in this sport. The development of pitch technology and major rule changes in recent years have meant that the previous research might not apply to modern play. 


\section{Research aims:}

The aim of current study was to analysis the scored goals during field hockey matches in 2014 Men's Hockey World Cup and design specific training program for improving the shooting skills toward the goal, through the following aims:

(1) Determine the shooting circle areas, which commonly used for shooting toward the goal in field hockey matches.

(2) Determine the most shooting skills, which used for scoring goals during the matches in field hockey.

(3) Design specific training program for improving the accuracy of shooting skills toward the goal in field hockey.

\section{Materials and Methods}

\section{Research curriculum}

The researchers used the experimental approach for current study by on group and have used the video analysis for analyzing the total scored goals in 2014 Men's Hockey World Cup.

\section{Subject}

Twenty players from Sharkia hockey club in Zagazig city participated in this study and registered in Egyptian field hockey federation. All players had at least 8 years of field hockey experience and competed in the Egyptian hockey championship less than 17 years. The players were currently participating in a hockey competition, and were free from any injury or illness. The homogeneity descriptive data of age, height, body mass, body mass index and training experience are presented in (Table 1).

\begin{tabular}{l|c|c|c|c}
\hline \multicolumn{1}{c|}{ Variables } & Average & SD & Median & Skewness \\
\hline Age $($ year $)$ & 15.85 & 0.59 & 16.00 & -0.77 \\
\hline Height $(\mathrm{m})$ & 1.71 & 0.04 & 1.72 & -0.21 \\
\hline Weight $(\mathrm{kg})$ & 62.50 & 4.27 & 62.05 & 0.32 \\
\hline BMI $\left(\mathrm{kg} / \mathrm{m}^{2}\right)$ & 21.34 & 1.57 & 21.12 & 0.43 \\
\hline TE (year) & 8.10 & 0.85 & 8.00 & 0.35 \\
\hline $\begin{array}{l}\text { SD = Standard deviation, BMI } \\
\text { experience Body mass index and TE }=\text { Training }\end{array}$
\end{tabular}


The data in (Table. 1) demonstrate the descriptive homogeneity of subject in anthropometric variables and training experience years of players. The skewness fall between $( \pm 3)$ and were $(-0.77$ to 0.35$)$, which refer to a homogeneity between all players.

\section{Procedures}

Prior to each testing session, subjects completed a standard warm-up that consisted of a 5 to 10 minutes jog, a series of stretches, and a sequence of 20 meter running drills. Each skill test was separated by at least a 5 minutes recovery period, but players were allowed more rest if they so required and completed the series of tests in a similar order. Players attended three testing sessions, test session (1) involved anthropometric measurements including standing height, body weight and the complex shooting accuracy test. Test session (2) involved three separated skills shooting accuracy tests, which were hitting, reverse hitting and pushing. Test session (3) involved the other three separated skills shooting accuracy tests, which were flick, slap shooting and deflection skill from two sides (right and left).

\section{(1) Video analysis}

In order to obtain the data information's about total scored goals in 2014 Men's Hockey World Cup, and how players shooting the balls toward the goal, and which shooting skills they used. The researcher collected the total scored goals videos form the international field hockey federation website (https://www.fih.ch).

After downloading the total scored goals videos, the (GeoGebra) software (https://www.geogebra.org) used for investigate the shooting circle areas, which commonly used for shooting toward the goal in Hockey World Cup matches.

The scouting skills analysis method used to investigate the most shooting skills, which used to scoring goals during the World Cup matches. The researcher has analyzed all scored goals in the tournament matches ( 28 matches) of the best 4 teams, which were played in the semi-final phase. 


\section{(2) Skills shooting accuracy tests}

\section{Complex shooting accuracy test}

Complex shooting accuracy test (Figure 1,2) was evaluated by placing a hockey ball at seven positions in the goal scoring area (shooting circle "D"). Players performed a series of four hits (shots 1 - 4), two pushes (shots 5 - 6), two flicks (shots 7 - 8), two slaps (shots 9 - 10), two deflection from right side (balls 11 12), and deflection left side (balls 13 - 14).

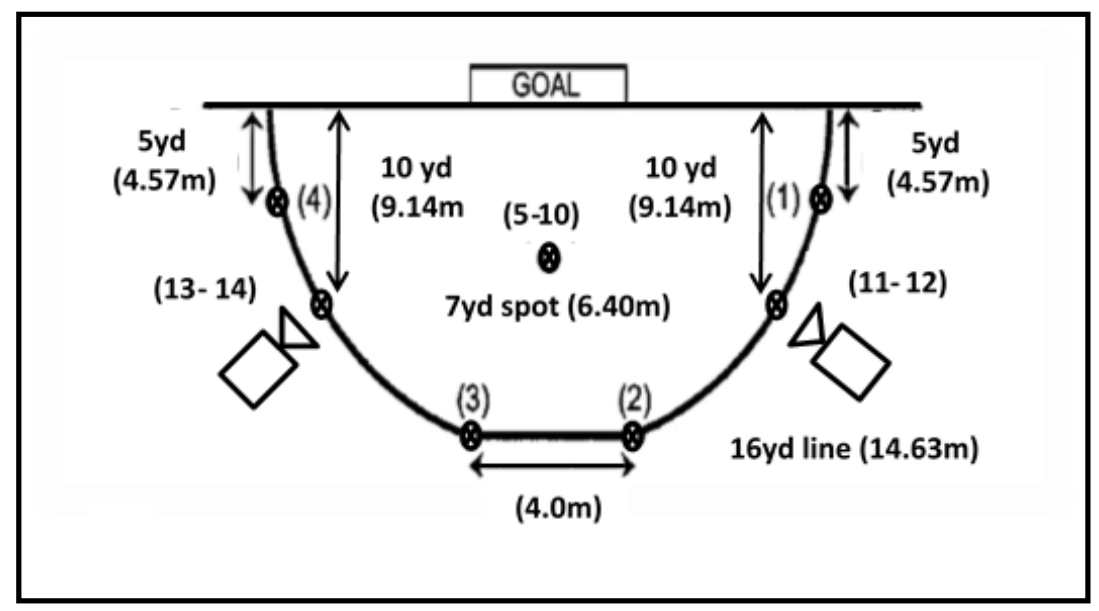

Figure 1. Schematic of complex shooting accuracy test

A flick is similar to a pushing action. However, in executing a flick, the player angles the stick in relation to the ball so that the ball undergoes both horizontal and vertical displacement. The result of each shot was recorded, with each accurate shot being awarded 1 point and each inaccurate shot 0 points. An accurate shot was defined as one that was hit into the target zone. If the shot hit the post, it was deemed accurate only if it rebounded into the goal. A score out of 14 was recorded for each player, with each player's performance then expressed as the percentage of accurate shots. As only shooting accuracy was assessed in this test, no special pushing, hitting, or flicking speed was required. Thus the accuracy component of goal scoring could be isolated from the power component, in accordance with the speed/accuracy tradeoff (Keogh et al., 2003; Spirduso \& Choi, 1993). 


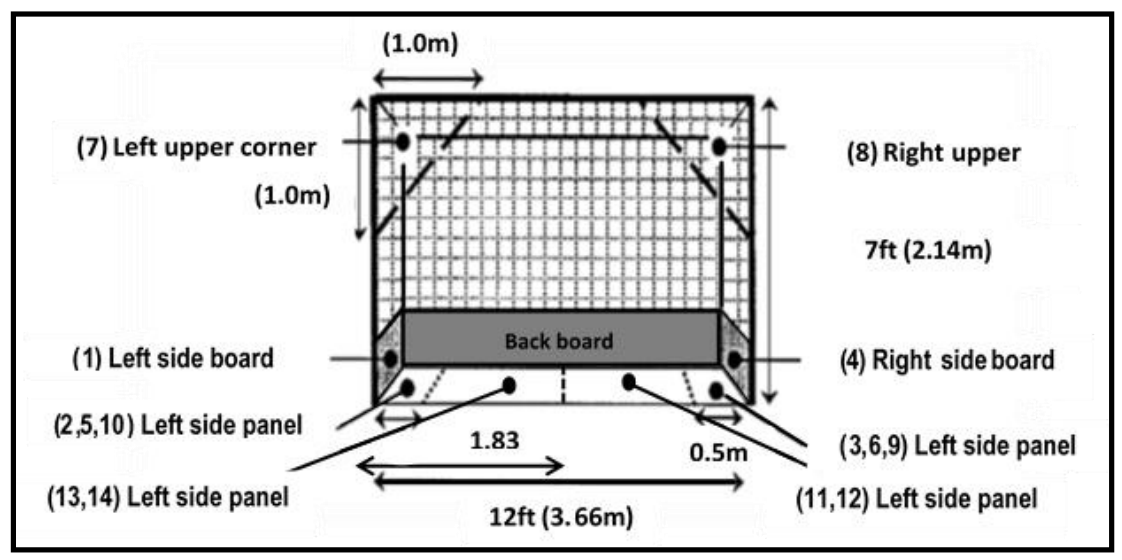

Figure 2. Schematic of goal scoring targets

\section{Separated skill shooting accuracy tests}

Separated skill shooting accuracy tests (Figure 3 and 2) was evaluated by placing a hockey ball at eight positions in the goal scoring area (shooting circle “D”). Players performed this test for 6 skills (hitting - reverse hitting - push flick - slap shot - deflection right and lift) separately. These skills selected based on match analysis in 2014 Men's Hockey World Cup, which recorded the highest rates during a shooting from goal scoring area on the target of the goal. A series of four hits (shots 1 - 4) were from the 6.40 meter and the other four hits (shots 5 - 8) were from the goal scoring area line (shooting circle "D"). The deflection skill test was evaluated from both sides (right and left) on one series by field hockey balls launcher, which has been thrown 4 balls from each side. Description of points score is similar to the complex shooting accuracy test and based on the schematic of goal scoring targets in (Figure 2). This battery accuracy test was developed and established by study of (Keogh et al., 2003); the validity and reliability were tested and the correlation coefficient was recorded by $(r=0.92)$ at confidence of ( $p$-value $\leq 0.05)$. 


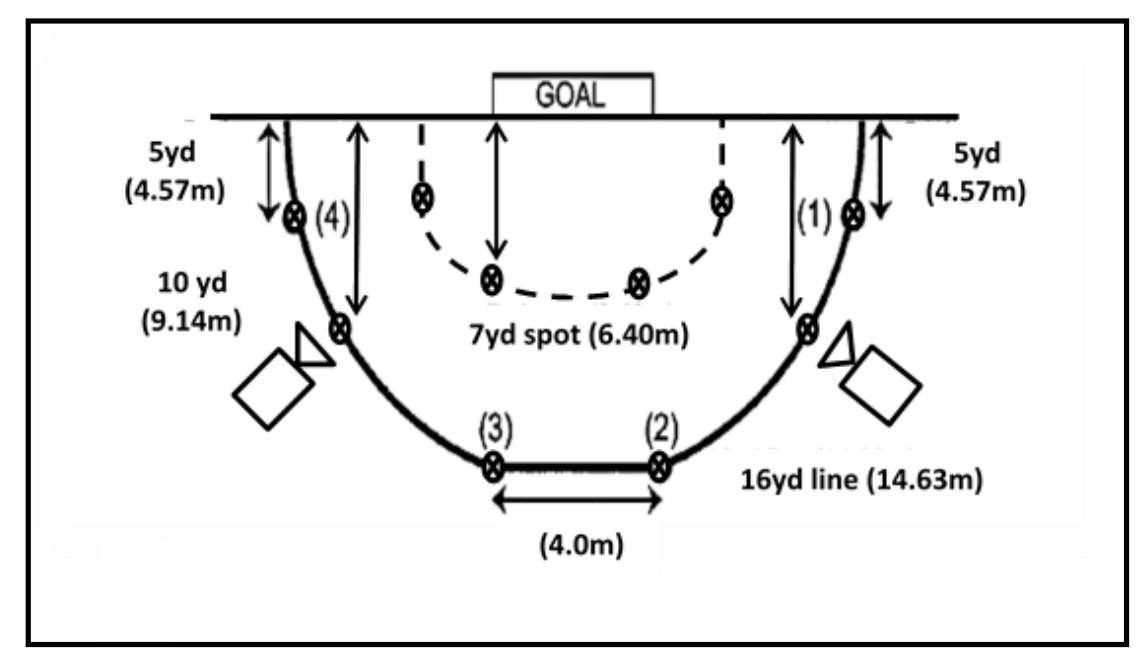

Figure 3. Schematic of complex shooting accuracy test

The view of schematic shooting accuracy test in (Figure 1, 2 and 3) demonstrates that, 4 circles indicate the position from which the shots were taken. Numbers in parentheses indicate each of the 8 shots taken. Shots $(1-4)=$ hits; Shots $(5-6)=$ pushes; Shots $(7-8)=$ flicks. Frontal view of the goal used in the shooting accuracy test. Numbers in parentheses indicate the target zone of each shot taken.

\section{Training program}

Shooting skills training was undertaken three sessions per week for 10 weeks and every session was between 45 - 60 minutes. The specific skill program was a part of the basic training program of players, which consists of 5 sessions and the session time is 120 minutes. The session in current study training program commenced with an identical 15 minutes warm up followed by 5 minutes specific warm up with ball. The exercise drills were completed within half playing field dimensions. The aim of each drill was to improve the accuracy of shooting on the goal targets and advices players about technical tips of shooting skills.

All drills selected were familiar to the players as they represented typical field hockey training skill drills in relation to drill duration and content, the specific training program concentrate on the shooting from different goal scoring "D" 
areas. The corresponding shooting drills were completed for the same duration as planed in the training program. Every training session consists of $3-4$ shooting drills. The type of exercise drills that each group performed was matched by intensity and, where possible, by similar drills. The objective of all skill drills was to develop the shooting accuracy without any active defense from players. The intensity of sessions was between 80 to $90 \%$ during the 3 sessions and the training skill drills was in the preseason period and in the last 10 weeks of the basic training program of the team.

\section{Statistical analysis}

Standard descriptive statistics (mean and standard deviation) were calculated for each variable. Normality of distribution of residuals was tested by means of the Kolmogorov-Smirnov test. The independent t-test was computed to compare significant differences between pre and posttests in selected skills measurements. Statistical significance was accepted at an alpha level of ( $p$-value $\leq 0.05$ ). All statistical tests were performed by SPSS statistical software 16.0 (Chicago, IL).

\section{Results}

The means \pm standard deviations (SD) results of complex shooting accuracy test and separated skill shooting accuracy tests at pretest and posttest are outlined in (Table. 2).

\begin{tabular}{|c|c|c|c|c|c|}
\hline Variables & Pre-Test & Post-Test & MD & t-statistic & p-value \\
\hline \multicolumn{6}{|c|}{ Complex shooting accuracy test } \\
\hline TS balls $(\mathrm{Nr})$ & $4.35 \pm 1.31$ & $9.15 \pm 0.88$ & 4.80 & 13.634 & $0.00 *$ \\
\hline \multicolumn{6}{|c|}{ Separated skill shooting accuracy tests } \\
\hline Push TSB (Nr) & $4.90 \pm 1.12$ & $6.05 \pm 0.76$ & 1.15 & 3.803 & $0.00 *$ \\
\hline Flick TSB (Nr) & $2.55 \pm 1.00$ & $4.15 \pm 0.88$ & 1.60 & 5.389 & $0.00 *$ \\
\hline Slap Shot TSB (Nr) & $3.45 \pm 1.10$ & $4.75 \pm 0.79$ & 1.30 & 4.302 & $0.00 *$ \\
\hline Hitting TSB $(\mathrm{Nr})$ & $3.65 \pm 1.18$ & $5.15 \pm 1.09$ & 1.50 & 4.173 & $0.00 *$ \\
\hline Reverse hitting TSB (Nr) & $2.25 \pm 0.79$ & $3.85 \pm 1.10$ & 1.60 & 5.326 & $0.00 *$ \\
\hline Deflection right TSB (Nr) & $1.85 \pm 0.75$ & $3.45 \pm 0.89$ & 1.60 & 6.176 & $0.00 *$ \\
\hline Deflection Left TSB (Nr) & $1.40 \pm 0.60$ & $2.65 \pm 0.75$ & 1.25 & 5.850 & $0.00 *$ \\
\hline
\end{tabular}

The results of (Table. 2) demonstrate significant difference between pretest and posttest data of field hockey players in total scored balls at $(p \leq 0.05)$ in shooting 
skills during both accuracy tests and the statistics data confirmed that t-statistic values were greater than t-critical value by 38 degree freedom.

The percentage means \pm standard deviations (SD) results of total scored balls (TSB) during accuracy tests at pretest and posttest are outlined in (Table. 3).

\begin{tabular}{l|c|c|c}
\hline \multicolumn{1}{c|}{ Variables } & Pre-Test & Post-Test & Improvement (\%) \\
\hline \multicolumn{4}{c}{ Complex shooting accuracy test } \\
\hline TS balls (\%) & $31.07 \pm 9.35$ & $65.36 \pm 6.25$ & $34.29 \%$ \\
\hline \multicolumn{4}{|c|}{ Separated skill shooting accuracy tests } \\
\hline Push TSB (\%) & $61.25 \pm 13.10$ & $75.63 \pm 9.49$ & $14.38 \%$ \\
\hline Flick TSB (\%) & $31.88 \pm 12.48$ & $51.88 \pm 10.94$ & $20.00 \%$ \\
\hline Slap Shot TSB (\%) & $43.13 \pm 13.74$ & $59.38 \pm 9.83$ & $16.25 \%$ \\
\hline Hitting TSB (\%) & $45.63 \pm 14.78$ & $64.38 \pm 13.62$ & $18.75 \%$ \\
\hline Reverse hitting TSB (\%) & $28.13 \pm 9.83$ & $48.13 \pm 13.62$ & $20.00 \%$ \\
\hline Deflection right TSB (\%) & $23.13 \pm 9.31$ & $45.28 \pm 11.09$ & $22.15 \%$ \\
\hline Deflection Left TSB (\%) & $17.50 \pm 7.48$ & $33.13 \pm 9.31$ & $15.63 \%$ \\
\hline MD = mean difference between both tests, TSB = Total Scored Balls \\
\hline
\end{tabular}

Likewise, the results in (Table. 3) demonstrate that the percentages of total scored ball from total shooting numbers on the goal targets in posttest were higher than pretest in the both shooting accuracy tests. In addition, the results demonstrate an improvements percentage in accuracy tests, which ranged between $14.38 \%$ in pushing TSB to $34.29 \%$ in TSB of complex shooting accuracy test.

\section{Discussion}

The perception of the skills requirements of field hockey players is required for successful performance in competitive. There are a wide number of previous studies have investigated some of anthropometric, physiological, technical and tactics characteristics of field hockey players, but no enough studies examined the important shooting skills, when players hits the ball in goal from the goal scoring area.

The purpose of current study were to identify the most hitting circle area which commonly used for hitting toward the goal target based on the match analysis during the 2014 Men's Hockey World Cup. In this context, the match analysis scouting data confirmed that, the $50 \%$ of the total shoots in the game has been 
hitting from the middle-left area, 34.7\% middle-right area, $8.3 \%$ left area and $2.8 \%$ right area.

The results of current study (Table. 2, 3) showed that, the special shooting skill drills in the training program were improved the shooting ability on the goal targets area, which reported percentage of $65.36 \%$ in the posttest of complex shooting accuracy test when compared to the mean percentage of $31.07 \%$ in the pretest and recorded improve percentage of $34.29 \%$ for the total scored balls from this areas. These results showed that the shooting accuracy improved by training program, which concentrate on the hockey drills from this areas when players shooting on the goal, and consisted with the study of (Keogh et al., 2003; Starkes, 1997; Thomas et al., 2009), who reported that skills of players during match gamed developed by special skills drills in training programs in sports.

The second objective in current study was to determine the most hitting skills to score goals during field hockey game. In this context, the match analysis of 4 best ranked teams in the world cup reported that, the most important used skills to score goals in the game were front hit (28.2\%), reverse hit (25.6\%), deflection (15.4\%), push (10.3\%), slap shot (7.7\%) and flick (5.1\%), respectively.

The results of current study (Table. 2, 3) showed that, the posttest values more accurate than the pretest values in the separated skill shooting accuracy tests, which reported percentage improvements of skill tests about $14.38 \%$ to $22.15 \%$. This finding may be explained by the drills in training that contained some tasks, which forcing the players to run and use the most important skills when shooting on the goal and as regarding the results of the match analysis. For example, it was used some training tools to perform the skill tasks to shooting the balls on the special targets, these tools such as the pylons and the design of special targets in the goal to simulate the shooting from the goal scoring area. This findings consisted with studies by (Lago-Peñas et al., 2010; Sunderland et al., 
2005), who suggested that the selected skills of players play an important role in winning and losing game.

The third aim current study was to develop the accuracy of hitting skills toward the goal from goal scoring area. The results of current study reported high accurate performance in the posttest compared to the accuracy values in the pretest. The findings in (Table 2, 3) confirmed that, the number of total shots on the goal target was increased after the training program; mean of complex shooting accuracy test score at posttest was 9.15 shots with mean difference 4.80 shots from the pretest and recorded an improvement percent of $34.29 \%$, this results confirmed the study of (Sofwan et al., 2012), who reported that the position of the player can effect on the types of shot chosen then on.

In addition, the current study found that push and hit shots score in separated skill shooting accuracy tests were higher in total numbers of scored goals, however all other means of other skills were significant difference between pre and posttests. This finding may be explained by the technics of push and hit skills; the push shot is when moving the ball along the ground by using a pushing movement of the stick, both the head of the stick and the ball are usually in contact with the ground during the pushing movement and the push shot can be execute from direct shot, pass and deflect.

In other hand, the hit shot is striking the ball using a swinging arm action movement of the stroke towards the ball, usually the hit shot involves power and produces speed to the ball velocity which gives a big deal for the keeper to see the ball clearly with the attempt to save the shot.

Finally, the training program generally was an effective on increasing the number of total shots, which scored from the goal scoring area. In addition, there is an important consideration that were control in drills during field hockey training such as some special skills that will be effective when player shot on the goal target and score a goal in match play. Moreover, the designed drills in 
training program with wide variety in shooting skill kinds have been increased the scores and percentage in posttest when compared to scores in pretest.

These findings of current study consist and confirmed the previous results in this research field (Farrow et al., 2008; T. J. Gabbett et al., 2010; T. Gabbett et al., 2009; Ortega et al., 2009; Vaz et al., 2010), which had a good attention toward developing the related skills during match play and the using of some variation training program.

\section{Conclusions}

The current study conclude that most favorable area when field hockey players shooting in the goal on the middle-left penetration of the goal scoring area "D". The most shots on the goal from penetration of the " $\mathrm{D}$ " were push and hit skills. The type of shots it seems that hit and push shot coming from middle right and left area is the most popular choice shot and area penetrated for the winning team. Field hockey coaches must be taken in their consideration the effective shooting skills in training drills for developing the training plan for team and individuals characteristics that suits this type of game play.

\section{References}

Abernethy, B. (1996). Training the visual-perceptual skills of athletes. Insights from the Study of Motor Expertise. Am J Sports Med, 24(6 Suppl), 89-92. Appleby, B., \& Dawson, B. (2002). Video analysis of selected game activities in Australian Rules Football. J Sci Med Sport, 5(2), 129-142.

Elferink-Gemser, M. T., Visscher, C., Lemmink, K. A., \& Mulder, T. W. (2004). Relation between multidimensional performance characteristics and level of performance in talented youth field hockey players. J Sports Sci, 22(11-12), 1053-1063.

Farrow, Damian, Pyne, David, \& Gabbett, Tim. (2008). Skill and physiological demands of open and closed training drills in Australian Football. International Journal of Sports Science and Coaching, 3(4), 489-499. 
Gabbett, Tim J, Jenkins, David G, \& Abernethy, Bruce. (2010). Physiological and skill demands of 'on-side'and 'off-side'games. The Journal of Strength \& Conditioning Research, 24(11), 2979-2983.

Gabbett, Tim, Jenkins, David, \& Abernethy, Bruce. (2009). Game-based training for improving skill and physical fitness in team sport athletes. International Journal of Sports Science and Coaching, 4(2), 273-283.

Hughes, Mike. (2004). Performance analysis-a 2004 perspective. International Journal of Performance Analysis in Sport, 4(1), 103-109.

Hughes, Mike, \& Bartlett, Roger. (2002). The use of performance indicators in performance analysis. Journal of sports sciences, 20(10), 739-754.

Keogh, J. W., Weber, C. L., \& Dalton, C. T. (2003). Evaluation of anthropometric, physiological, and skill-related tests for talent identification in female field hockey. Can J Appl Physiol, 28(3), 397-409.

Lago-Peñas, Carlos, Lago-Ballesteros, Joaquín, Dellal, Alexandre, \& Gómez, Maite. (2010). Game-related statistics that discriminated winning, drawing and losing teams from the Spanish soccer league. Journal of sports science \& medicine, 9(2), 288.

Lames, Martin., \& Hansen, Gunnar. (2001). Designing observational systems to support top-level teams in game sports. International Journal of Performance Analysis in Sport, 1(1), 83-90.

Lemmink, K. A., Elferink-Gemser, M. T., \& Visscher, C. (2004). Evaluation of the reliability of two field hockey specific sprint and dribble tests in young field hockey players. Br J Sports Med, 38(2), 138-142.

Lythe, John. (2008). The physical demands of elite men's field hockey and the effects of differing substitution methods on the physical and technical outputs of strikers during match play. Auckland University of Technology.

Ortega, Enrique, Villarejo, Diego, \& Palao, José M. (2009). Differences in game statistics between winning and losing rugby teams in the Six Nations Tournament. Journal of sports science \& medicine, 8(4), 523. 
Sofwan, N, Norasrudin, S, Redzuan, P, \& Mubin, A. (2012). Distinguishing Playing Pattern between Winning and Losing Field Hockey Team in Delhi FIH Road to London 2012 Tournament. Paper presented at the Proceedings of World Academy of Science, Engineering and Technology. Spencer, M., Lawrence, S., Rechichi, C., Bishop, D., Dawson, B., \& Goodman, C. (2004). Time-motion analysis of elite field hockey, with special reference to repeated-sprint activity. J Sports Sci, 22(9), 843-850.

Spirduso, Waneen W, \& Choi, JongHwan. (1993). Age and practice effects on force control of the thumb and index fingers in precision pinching and bilateral coordination Sensorimotor impairment in the elderly (pp. 393412): Springer.

Starkes, Janet L. (1997). Skill in field hockey: The nature of the cognitive advantage. Journal of sport psychology, 9(2), 146-160.

Sunderland, C, Bussell, C, Atkinson, G, Kates, M, \& Alltree, R. (2005). Notational analysis of goals scored from open play in international field hockey. J Sports Sci, 23(11/12), 1300-1301.

Thomas, Camille, Fellingham, Gilbert, \& Vehrs, Pat. (2009). Development of a notational analysis system for selected soccer skills of a women's college team. Measurement in Physical Education and Exercise Science, 13(2), 108-121.

Vaz, Luis, Van Rooyen, Michele, \& Sampaio, Jaime. (2010). Rugby gamerelated statistics that discriminate between winning and losing teams in IRB and Super twelve close games. Journal of sports science \& medicine, 9(1), 51.

\section{Internet References}

http://www.fih.ch/

https://www.geogebra.org 